\title{
Effect of carbon and oxygen impurity atoms on the migration rate of tilt boundaries in fcc metals: a molecular dynamics simulation
}

\author{
G. M. Poletaev ${ }^{\dagger, 1}$, I. V. Zorya ${ }^{2}$, R. Y. Rakitin ${ }^{3}$, M. A. Iliina ${ }^{4}$, M. D. Starostenkov ${ }^{1}$ \\ †gmpoletaev@mail.ru \\ ${ }^{1}$ Altai State Technical University n. a. I. I. Polzunov, 46 Lenina Av., Barnaul, 656038, Russia \\ ${ }^{2}$ Siberian State Industrial University, 25 Bardin Av., Novokuznetsk, 654007, Russia \\ ${ }^{3}$ Altai State University, 61 Lenin Av., Barnaul, 656049, Russia \\ ${ }^{4}$ Financial University under the Government of the Russian Federation, 54 Lenin Av., Barnaul, 656036, Russia
}

\begin{abstract}
The effect of carbon and oxygen impurity atoms on the migration rate of tilt boundaries with the $\langle 100\rangle$ and $\langle 111\rangle$ misorientation axes in fcc metals $\mathrm{Ni}, \mathrm{Ag}, \mathrm{Al}$ was studied by means of the molecular dynamics method. It is shown that the introduction of impurity atoms of light elements led to a significant inhibition of the migration of grain boundaries: with the introduction of $5 \%$ by almost an order of magnitude, $10 \%$ - by two orders of magnitude. Carbon atoms tend to form aggregates, which, being fixed on the grain boundary, become effective stoppers that prevent the boundary moving. Oxygen atoms did not form aggregates, but because of the high values of the binding energy with the boundaries, they also effectively hampered their migration. In contrast to the formation of aggregates by carbon atoms, in the case of oxygen impurity, another effect takes place - "loosening" and an increase in the width of the boundary. For impurity atoms of carbon and oxygen, the binding energies with grain-boundary edge dislocations in the metals under consideration were calculated. The obtained values correlate well with the dependences of the grain boundary migration rate on the impurity concentration: the greatest effect of impurities on the boundary migration rate and the value of the binding energy were obtained for the Al-C system, the smallest - for Ag-O.
\end{abstract}

Keywords: molecular dynamics, grain boundary, migration, tilt boundary, impurity.

\section{Introduction}

Grain boundary migration is the movement of the boundary along the normal to its surface. Migration plays a decisive role in the process of recrystallization and in many instances of the phase transformations. Despite the long-standing interest in the problem of migration of grain boundaries, there are still disagreements and unresolved issues. It is believed that low-angle tilt boundaries migrate through the combined action of two mechanisms: slip and climb of grain boundary dislocations [1]. In [2,3], for example, the authors come to the conclusion that the main mechanism of migration of the tilt boundaries is the climbing of grain boundary dislocations. The cooperative climb of dislocations was likely took place during high-speed migration of symmetric boundaries, discovered by the authors of [4] in a computer model when shear stresses along the boundary were created in a sample.

On the other hand, the results of $[5,6]$, on the contrary, indirectly support the predominance of the slip mechanism of grain-boundary dislocations. In $[7,8]$, we have studied the atomic mechanism of migration of tilt boundaries with the $<100>$ and $<111>$ misorientation axes, as well as their triple junctions, using the molecular dynamics method. It is shown that the migration and elongation of the low-angle $<100>$ boundaries is carried out by splitting of the paired grain boundary dislocations with the subsequent change of the dislocations-partners. The dislocations-partners are replaced through the slip of split dislocations. The migration of the $<111>$ tilt boundary occurred as a result of the combined action of two mechanisms: the mechanism described above and the mechanism consisting in the joint slipping of pair grain-boundary dislocations, which, in contrast to grainboundary dislocations in the $<100>$ boundaries, have common slip planes.

Another important issue is the effect on the mobility of the grain boundaries of impurities, lattice defects, free volume. In [9], we have considered the effect of hydrogen impurity on the migration mobility of the tilt boundaries in palladium. It was shown that when the hydrogen concentration in palladium is $50 \%$, the rate of migration of the boundaries slows down about two times. For low-angle boundaries, the binding energy is obviously close to the binding energy of impurity atoms with dislocations. In $[10,11]$, for example, it were obtained the values of the binding energy of a carbon atom with a dislocation in iron in the range of $0.4-0.7 \mathrm{eV}$; in [12], for oxygen atom in zirconium $-0.5 \mathrm{eV}$. The order of magnitude indicates a fairly high bind of impurity atoms with both dislocations and grain boundaries. It is known that grain boundaries, as well as individual dislocations, collect around it an atmosphere of impurities, similar to the Cottrell atmosphere [13]. The migration mobility of boundaries in this case is significantly reduced, since, as in the case of dislocations, additional energy is required to detach the boundary from the atmosphere. 
This paper is devoted to the study of the influence of carbon and oxygen impurity atoms on the rate of migration of tilt boundaries with the $<100>$ and $<111>$ misorientation axes in $\mathrm{fcc}$ metals $\mathrm{Ni}, \mathrm{Ag}, \mathrm{Al}$. This set of three metals is unique in that two of them have almost the same radii of atoms, while the other two have almost identical electronegativities. The radii of atoms: $\mathrm{Al}-1.43 \AA$, Ag $-1.44 \AA ̊ \mathrm{~N}, \mathrm{Ni}-1.24 \AA$ [14]. Electronegativity (Pauling scale): $\mathrm{Al}-1.61, \mathrm{Ag}-1.93, \mathrm{Ni}-1.91$ [15].

\section{Description of the model}

The model in the present work is based on the technique for studying the migration of tilt grain boundaries, proposed and developed in $[1,16]$. A clearly defined boundary was created in the form of a half-loop or arch, as in Fig. 1 (black dotted line). The tension of the boundary, which, like surface tension, arises from the tendency of the boundary to minimize its energy, is the reason for the directed movement of the boundary at the top part of the "half-loop" toward a decrease in its area. The force that provokes the migration and the boundary migration velocity remains constant throughout almost the entire movement of the boundary, smoothly decreasing by the end of the computer experiment.

The computational cell in the molecular dynamics model was created in the form of a plate 12 atomic layers thick (Fig. 1). The cells contained approximately 50000 atoms and had a height of $18.0 \mathrm{~nm}$, a width of $12.0 \mathrm{~nm}$, and a thickness of $2.4 \mathrm{~nm}$. Along the Z-axis (Fig. 1), an infinite repetition of the structure was simulated, i.e. the periodic boundary conditions were imposed. At the edge of the computational cell, the grain boundaries must be fixed, which implies preserving the orientation of the crystal lattice of two different grains at the border of the cell. In this connection, along the $\mathrm{X}$ and $\mathrm{Y}$ axes, the block borders (highlighted in dark gray in Fig. 1) were rigidly fixed to preserve the given misorientation of the grains.

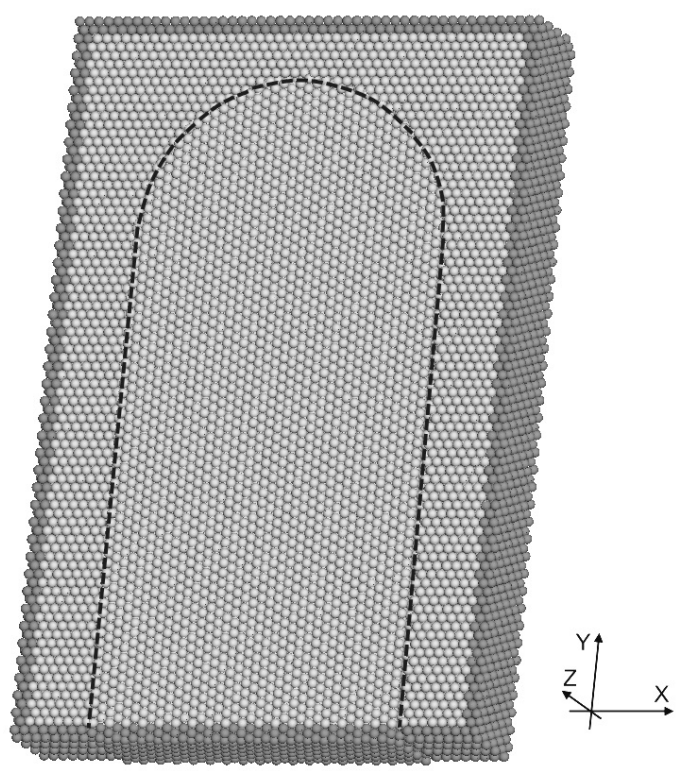

Fig. 1. Computational cell for modeling the migration of $<111>30^{\circ}$ tilt boundary. The dark-gray atoms on the edge of the computational cell remained motionless during the computer experiment (rigid boundary conditions).
The interactions of metal atoms with each other were described by the tight-binding Cleri-Rosato potentials [17]. To describe the interactions of impurity atoms of light elements with metal atoms and impurity atoms with each other, the Morse potential was chosen. Cleri-Rosato and Morse potentials have proved themselves in numerous calculations performed by the molecular dynamics method [18-20]. The parameters of the potentials for describing the interactions of impurity atoms $\mathrm{C}$ and $\mathrm{O}$ with the metal atoms under consideration were taken from [21], where they were found taking into account empirical dependencies and known characteristics, such as the melting or decomposition temperature of the corresponding chemical compound of a metal with a light element, activation energy of the diffusion of an impurity atom in the crystal lattice of the metal. In [21], to describe the interactions of impurity atoms with each other in metals, the potentials proposed by other authors have been taken as a basis. For the C-C bond, the pair potential from [22] was transformed into Morse potentials. For O-O bonds, potentials were taken from [23].

Impurity atoms were introduced into the computational cell containing the grain boundary randomly into octahedral voids. As is well known, impurity atoms of light elements, such as carbon and oxygen, are located in the fcc lattice of metals in octahedral sites [9]. Simulation of the migration of grain boundaries was carried out at a temperature of $0.95 \cdot T_{m}$, where $T_{m}$ is the melting point. The choice of a temperature almost equal to the melting point is due to the fact that at this temperature the highest rate of grain boundary migration is observed. The temperature in the model was set through the initial velocities of the atoms according to the Maxwell-Boltzmann distribution, wherein the thermal expansion of the calculation blocks was taken into account. To keep the temperature constant during the simulation, the Nose-Hoover thermostat was used.

\section{Results and discussion}

The introduction of carbon and oxygen impurity atoms led to a significant inhibition of the migration of grain boundaries, which is obviously due to the high binding energies of impurity atoms with them. Carbon atoms at a relatively high concentration in the metal (more than $5 \%$ ) in the course of the computer experiment sought to form aggregates that mainly concentrated at the grain boundary (Fig. 2 a). These aggregates turned out to be effective stoppers for the moving boundary - their formation led to a decrease in the rate of migration of the grain boundary by one to two orders of magnitude. The aggregates were formed at the initial stage of modeling and they contained from several carbon atoms to two dozen. During the entire simulation, they, as a rule, remained motionless, including when the migrated boundary passed through them.

Oxygen atoms repel each other in a metal, in contrast to carbon atoms, which tend to form bonds [12]. Oxygen atoms did not form aggregates in simulating the migration of grain boundaries, but because of the high values of the binding energy with the boundaries, they also effectively hampered their migration. In the case of oxygen impurity, another effect 
was observed - "loosening" and an increase in the width of the boundary (Fig. 2b). An additional disordering of the structure occurred, in some cases even with the formation of an amorphous region behind the migrating boundary. The presence of oxygen atoms, apparently, leads to the difficulty in forming a crystalline structure behind the migrating high-angle boundary. As mentioned above, the top part of the "half-loop" migrates more intensively than the lateral boundaries; therefore, it is more blurred in Fig. 2 b.

Fig. 3 shows the dependences of the migration rate of the $<100>$ and $<111>$ tilt boundaries with a misorientation angle of $30^{\circ}$ at a temperature of $0.95 \cdot T_{m}$ on the concentration of impurity atoms. The migration rate, according to the method described in $[1,16]$, was calculated from the boundary displacement at the top part of the "half-loop". The duration of the molecular dynamics experiments ranged from several hundred to several thousand picoseconds.

As can be seen from the above graphs, the migration rates of similar boundaries in pure metals $\mathrm{Ni}, \mathrm{Ag}$, and $\mathrm{Al}$ have close values at different temperatures $(1640 \mathrm{~K}, 1173 \mathrm{~K}$, and $887 \mathrm{~K}$ respectively), but with the same ratio to the melting point of the metal, approximately equal to $0.95 \cdot T_{m}$. A similar connection of various activation processes with the melting point of metals was repeatedly noted by different authors $[1,24]$.

The boundaries with the misorientation axis $<111>$, as can be seen in Fig. 3, migrate approximately two times faster at the temperature under consideration than the $<100>$ boundaries. The same result was obtained in [8].

Impurity atoms of carbon and oxygen, according to the data obtained, strongly inhibit the migration of grain boundaries - with the introduction of $5 \%$ by almost an order of magnitude, $10 \%$ - by two orders of magnitude. Moreover, for $\mathrm{Al}$ at concentrations of carbon atoms above $5 \%$, it was not possible to measure the rate of movement of the boundary even for relatively long computer experiments (a few ns), the boundary remained in place.

The influence of impurities on the migration of grain boundaries in $\mathrm{Ag}$ was the smallest compared to $\mathrm{Ni}$ and $\mathrm{Al}$, which, as it turned out, correlates well with the binding energy of impurity atoms with edge grain-boundary dislocations (Table 1).

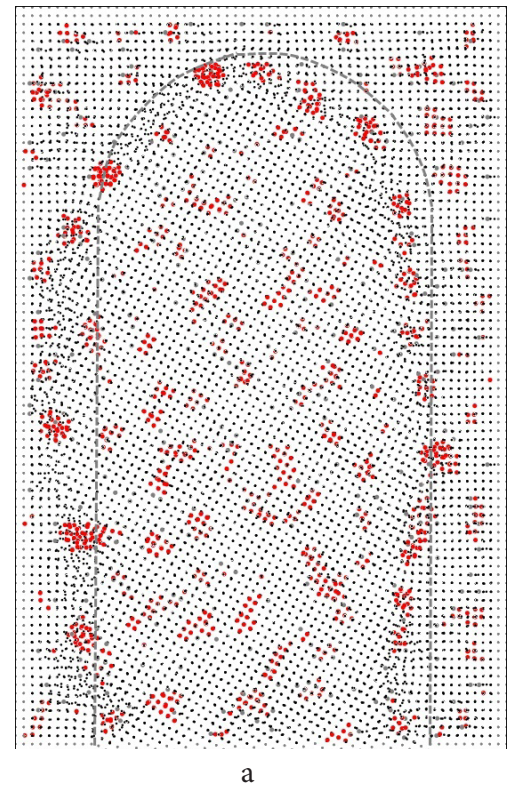

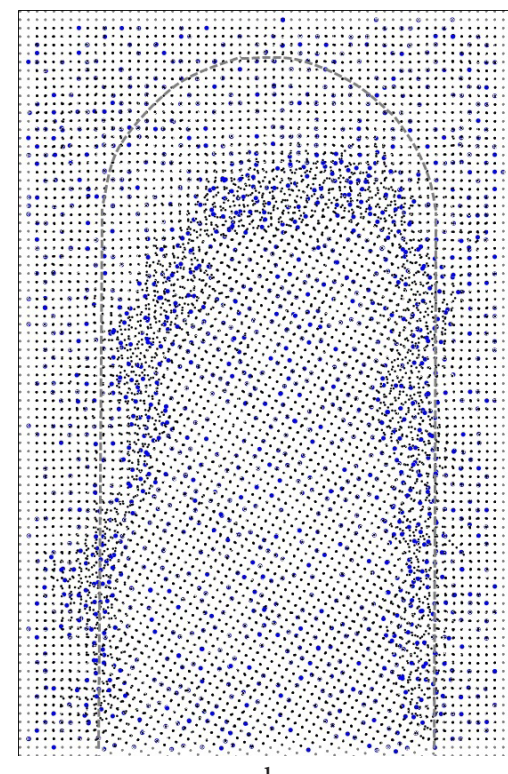

$\mathrm{b}$

Fig. 2. (Color online) The formation of aggregates of carbon atoms (a) and "loosening" of the grain boundary due to the presence of impurity oxygen atoms (b) in $\mathrm{Ni}$ when simulating the migration of $<100>30^{\circ}$ tilt boundaries. Impurity carbon atoms are red, oxygen - blue. The gray dashed line shows the initial position of the boundary. Figures are obtained after cooling the computational cell.

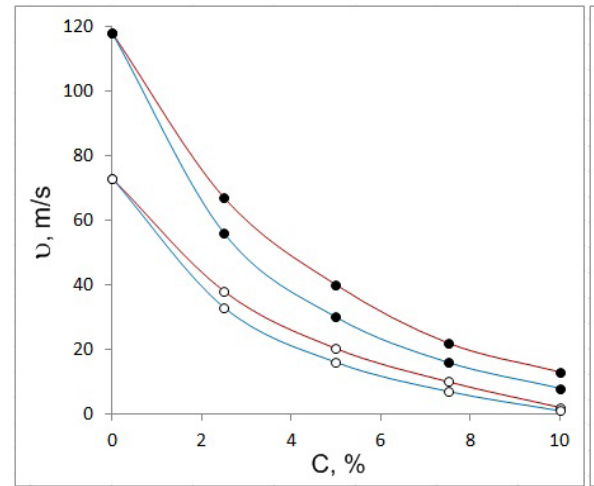

a

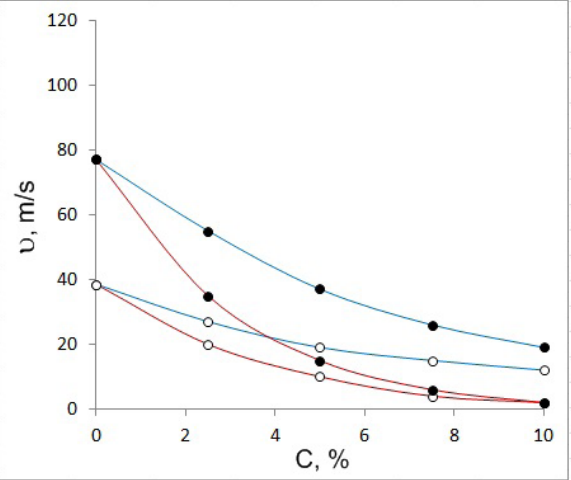

b

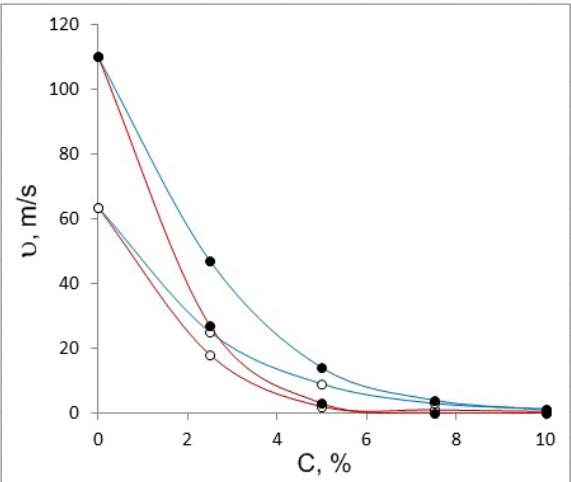

c

Fig. 3. (Color online) Dependencies of migration rate of $\langle 111\rangle$ (black markers) and $\langle 100\rangle$ (white markers) tilt boundaries with a misorientation angle of $30^{\circ}$ at a temperature of $0.95 \cdot T_{m}$ on the concentration of carbon (red curves) and oxygen (blue curves) impurity atoms: in $\mathrm{Ni}(\mathrm{a})$; in $\mathrm{Ag}(\mathrm{b})$; in $\mathrm{Al}$ (c). 
Table 1. The binding energy of carbon and oxygen atoms with the edge grain-boundary dislocation $(\mathrm{eV})$.

\begin{tabular}{|c|c|c|c|}
\hline & $\mathrm{Ni}$ & $\mathrm{Ag}$ & $\mathrm{Al}$ \\
\hline $\mathrm{C}$ & 0.77 & 0.30 & 1.34 \\
\hline $\mathrm{O}$ & 0.62 & 0.10 & 0.44 \\
\hline
\end{tabular}

The binding energy of an impurity atom with a grainboundary dislocation was calculated as the difference between the potential energy of the computational cell containing the low-angle tilt boundary and impurity atom in the octahedral site at such a distance from each other, which eliminates the interaction of dislocations and the impurity atom, and the potential energy of the computational cell containing impurity atom in the dislocation core. In both cases, before the calculation of the energy of the computational cell, the structure was relaxed, after which the computational cell was cooled to $0 \mathrm{~K}$. The position of the impurity atom in the dislocation core was chosen such that the binding energy was the highest, that is, the energetically most favorable position of the impurity in the dislocation was chosen.

\section{Conclusion}

The effect of carbon and oxygen impurity atoms on the rate of migration of tilt boundaries with the $<100>$ and $<111>$ misorientation axes in $\mathrm{fcc}$ metals $\mathrm{Ni}, \mathrm{Ag}, \mathrm{Al}$ was studied by mean of the molecular dynamics method. It was shown that the introduction of impurity atoms of light elements led to a significant inhibition of the migration of grain boundaries: with the introduction of $5 \%$ by almost an order of magnitude, $10 \%$ - by two orders of magnitude. Carbon atoms tend to form aggregates, which, being fixed on the grain boundary, become effective stoppers that prevent the boundary moving. Oxygen atoms did not form aggregates, but because of the high values of the binding energy with the boundaries, they also effectively hampered their migration. In contrast to the formation of aggregates by carbon atoms, in the case of oxygen impurity another effect takes place "loosening" and an increase in the width of the boundary.

For impurity atoms of carbon and oxygen, the binding energies with grain-boundary edge dislocations in the metals under consideration were calculated. The obtained values correlate well with the dependences of the grain boundary migration rate on the impurity concentration: the greatest effect of impurities on the boundary migration rate and the value of the binding energy were obtained for the Al-C system, the smallest - for Ag-O.

\section{References}

1. G. Gottstein, L.S. Shvindlerman. Grain Boundary Migration in Metals: Thermodynamics, Kinetics, Applications, 2nd edn. Boca Raton, CRC Press (2009) 711 p. $\underline{\text { Crossref }}$
2. R.W. Balluffi, J.W. Cahn. Acta Metallurgica. 29, 493 (1981). Crossref

3. M. Winning, A.D. Rollett, G. Gottstein, D. J. Srolovitz, A. Lim, L. S. Shvindlerman. Philosophical Magazine. 90, 3107 (2010). Crossref

4. K.P. Zolnikov, D.S. Kryzhevich, A.V. Korchuganov. Letters on Materials. 9 (2), 197 (2019). Crossref

5. Y. Huang, F. J. Humphreys. Acta Materialia. 47, 2259 (1999). Crossref

6. Y. Huang, F.J. Humphreys. Materials Chemistry and Physics. 132, 166 (2012). Crossref

7. G. Poletaev, I. Zorya, R. Rakitin. Computational Materials Science. 148, 184 (2018). Crossref

8. G. M. Poletaev, I.V. Zorya, M.D. Starostenkov, R. Yu. Rakitin, P. Ya. Tabakov. Journal of Experimental and Theoretical Physics. 128 (1), 88 (2019). Crossref

9. G.M. Poletaev, I.V. Zorya, M.D. Starostenkov, R. Yu. Rakitin, D. V. Kokhanenko. Russian Physics Journal. 61 (7), 1236 (2018). $\underline{\text { Crossref }}$

10. R. G. A. Veiga, H. Goldenstein, M. Perez, C. S. Becquart. Scripta Materialia. 108, 19 (2015). Crossref

11. L. E. Karkina, I. N. Karkin, I. L. Yakovleva, T. A. Zubkova. The Physics of Metals and Metallography. 114 (2), 155 (2013). Crossref

12. A. Atrens. Scripta Metallurgica. 8, 401 (1974). Crossref

13. V. Sursaeva, P. Zieba. Defect and Diffusion Forum. $237-240,578$ (2005). Crossref

14. H.J. Goldschmidt. Interstitial Alloys. London, Butterworths (1967) 640 p. Crossref

15. L. Pauling. The Nature of the Chemical Bond, 3rd edn. Ithaca, Cornell University Press (1960) 664 p.

16. S. G. Protasova, V.G. Sursaeva, L.S. Shvindlerman. Physics of the Solid State. 45, 1471 (2003). Crossref

17. F. Cleri, V. Rosato. Physical Review B. 48 (1), 22 (1993). Crossref

18. I. V. Zorya, G. M. Poletaev, R. Y. Rakitin, M. A. Ilyina, M.D. Starostenkov. Letters on Materials. 9 (2), 207 (2019). Crossref

19. G.M. Poletaev, I. V. Zorya, D.V. Novoselova, M.D. Starostenkov. International Journal of Materials Research. 108 (10), 785 (2017). Crossref

20. G.M. Poletaev, D. V. Novoselova, I. V. Zorya, M. D. Starostenkov. Physics of the Solid State. 60 (5), 847 (2018). Crossref

21. I. V. Zorya, G.M. Poletaev, M.D. Starostenkov. Fundamentalnye problemy sovremennogo materialovedenia. 15 (4), 526 (2018). (in Russian)

22. M. Ruda, D. Farkas, G. Garcia. Computational Materials Science. 45, 550 (2009). Crossref

23. P. Vashishta, R. K. Kalia, A. Nakano, J.P. Rino. Journal of Applied Physics. 103, 083504 (2008). Crossref

24. V.B. Vykhodets, T.E. Kurennykh, A.S. Lakhtin, A. Ya. Fishman. Solid State Phenomena. 138, 119 (2008). $\underline{\text { Crossref }}$ 